\title{
SHAPE FIBRATIONS AND FIBER SHAPE EQUIVALENCES, I
}

By

\author{
Hisao KaTo
}

\section{Introduction.}

In [6], Coram and Duvall introduced approximate fibrations and Mardešić and Rushing [11] generalized this and defined shape fibrations. For compact ANR's, shape fibrations agree with approximate fibrations. M. Jani, analogous to fiber maps, defined fiber morphisms and fiber shape equivalences [8]. In [4], Chapman proved the Complement Theorem, i.e., if $X$ and $Y$ are $Z$-sets in the Hilbert cube $Q$, then $X$ and $Y$ have the same shape (i.e., $\operatorname{Sh}(X)=\operatorname{Sh}(Y)$, see [2]) iff $Q-X$ and $Q-Y$ are homeomorphic.

In this paper, we define notions of fiber fundamental sequences and fiber shape equivalences and prove that if a fiber fundamental sequences between approximate fibrations is a shape equivalence, then it is a fiber shape equivalence. Also, we prove the following: Let $E, E^{\prime}$ and $B$ be compacta in the Hilbert cube $Q$ and let $E, E^{\prime} \subset Q$ be $Z$-sets. Then a map $p: E \rightarrow B$ over $B$ is fiber shape equivalent to a map $p^{\prime}: E^{\prime} \rightarrow B$ over $B$ if and only if there is a homeomorphism $h: Q-E \cong Q-E^{\prime}$ such that for each $b \in B$ and each neighborhood $W^{\prime}$ of $p^{\prime-1}(b)$ in $Q$, there is a neighborhood $W$ of $p^{-1}(b)$ in $Q$ such that $h(W-E) \subset W^{\prime}-E^{\prime}$.

All spaces considered will be metrizable. If $x$ and $y$ are points of a metric space, $d(x, y)$ denotes the distance from $x$ to $y$. A proper map $p: E \rightarrow B$ between locally compact, separable metric ANR's is an approximate fibration [6] if given an open cover $\mathcal{U}$ of $B$, a space $X$ and maps $h: X \rightarrow E, H: X \times I \rightarrow B$ such that $p h=H_{0}$, then there is a homotopy $\tilde{H}: X \times I \rightarrow E$ such that $\widetilde{H}_{0}=h$ and $H$ and $p \tilde{H}$ are $U$-close, where $H_{i}(x)=H(x, t)$. Let $\underline{E}=\left(E_{i}, q_{i j}\right)$ and $\underline{B}=\left(B_{i}, r_{i j}\right)$ be inverse sequences of compacta and let $\underline{p}=\left(p_{i}\right)$ be a sequence of maps $p_{i}: E_{i} \rightarrow B_{i}$. Then $\underline{p}: \underline{E} \rightarrow \underline{B}$ is a level $m a p$ if for any $i$ and $j \geqq i, p_{i} q_{i j}=r_{i j} p_{j}$. A map $p: E \rightarrow B$ between compacta is a shape fibration [11] if there is a level map $\underline{p}: \underline{E} \rightarrow \underline{B}$ of compact ANR-sequences with $\lim E=E, \lim B=B$ and $\lim _{\leftarrow} p=p$ satisfying the following property; for each $i$ and $\varepsilon>0$ there is $j \geqq i$ and $\delta>0$ such that for any space $X$ and any $h: X \rightarrow E_{j}$, $H: X \times I \rightarrow B_{j}$ with $d\left(p_{j} h, H_{0}\right)=\sup \left\{d\left(p_{j} h(x), H_{0}(x)\right) \mid x \in X\right\}<\delta$, there is a homotopy 
$\widetilde{H}: X \times I \rightarrow E_{i}$ such that $d\left(\widetilde{H}_{0}, q_{i j} h\right)<\varepsilon$ and $d\left(p_{i} \widetilde{H}, r_{i j} H\right)<\varepsilon$. Such $\left(E_{j}, \delta\right)$ is called a lifting pair for $\left(E_{i,}, \hat{)}\right)$.

\section{Fiber fundamental sequences.}

In [8], M. Jani introduced the notions of fiber morphisms and fiber shape equivalences. In this section, we conveniently give the following definitions (compare [8, Definition 4.1, 4.2 and 4.3]). It is assumed that $E, E^{\prime}$ and $B$ are compacta contained in the Hilbert cube $Q$ and maps $\tilde{p}: Q \rightarrow Q$ and $\tilde{p}^{\prime}: Q \rightarrow Q$ are extensions of maps $p: E \rightarrow B$ and $p^{\prime}: E^{\prime} \rightarrow B$, respectively.

Definition 1.1. A fundamental sequence (see [2]) $f=\left\{f_{n}, E, E^{\prime}\right\}_{Q, Q}$ is a fiber fundamental sequence over $B$ if for any $\varepsilon>0$ and any neighborhood $U^{\prime}$ of $E^{\prime}$ in $Q$ there is a neighborhood $U$ of $E$ in $Q$ and an integer $n_{0}$ such that for each $n \geqslant n_{0}$ there is a homotopy $H: U \times I \rightarrow U^{\prime}$ satisfying

(1) $H_{0}=f_{n_{0}} \mid U$ and $H_{1}=f_{n} \mid U$,

(2) $d\left(\tilde{p}^{\prime} H(x, t), \tilde{p}(x)\right)<\varepsilon, \quad x \in U, t \in I$.

REMARK 1.2. Definition 1.1 is independent of the choices of the extensions $\tilde{p}$ and $\tilde{p}^{\prime}$ of $p$ and $p^{\prime}$, respectively.

Definition 1.3. A fiber fundamental sequence $f=\left\{f_{n}, E, E^{\prime}\right\}_{Q, Q}$ over $B$ is fiber homotopic to a fiber fundamental sequence $g=\left\{g_{n}, E, E^{\prime}\right\}_{Q, Q}$ over $B(f \widetilde{B} g)$ if for any $\varepsilon>0$ and any neighborhood $U^{\prime}$ of $E^{\prime}$ in $Q$ there is a neighborhood $U$ of $E$ in $Q$ and an integer $n_{0}$ such that for any $n \geqq n_{0}$ there is a homotopy $K: U \times I \rightarrow U^{\prime}$ satisfying

(1) $K_{0}=f_{n} \mid U$ and $K_{1}=g_{n} \mid U$,

(2) $d\left(\tilde{p}^{\prime} K(x, t), \tilde{p}(x)\right)<\varepsilon, \quad x \in U, t \in I$.

REMARK 1.4. If $f: E \rightarrow E^{\prime}$ is a fiber map over $B$ (i.e. $p^{\prime} f=p$ ), $f$ induces a fiber fundamental sequence $f=\left\{f_{n}, E, E^{\prime}\right\}_{Q, Q}$, where $f_{n}=\tilde{f}: Q \rightarrow Q$ is an extension of $f$. Also, we can easily see that the composition of fiber fundamental sequences over $B$ is a fiber fundamental sequence over $B$.

Proposition 1.5. Let $p: E \rightarrow B, p^{\prime}: E^{\prime \prime} \rightarrow B$ and $p^{\prime \prime}: E^{\prime \prime} \rightarrow B$ be maps over $B$ and let $f_{i}=\left\{f_{i, n}, E, E^{\prime}\right\}_{Q, Q}$ and $g_{i}=\left\{g_{i, n}, E^{\prime}, E^{\prime \prime}\right\}_{Q, Q}(i=1,2)$ be fiber fundamental sequences over $B$. If $f_{1} \underset{B}{\widetilde{f}} f_{2}$ and $\underline{g}_{1} \underset{B}{\widetilde{g}} \underline{g}_{2}$, then $\underline{g}_{1} f_{1} \underset{B}{\widetilde{g}} \underline{g}_{2} f_{2}$.

Definition 1.6. A map $p: E \rightarrow B$ over $B$ is fiber shape equivalent to a map 
$p^{\prime}: E^{\prime} \rightarrow B$ over $B$ if there are fiber fundamental sequences over $B f=\left\{f_{n}, E, E^{\prime}\right\}_{Q, Q}$ and $g=\left\{g_{n}, E^{\prime}, E\right\}_{Q, Q}$ such that $g f \widetilde{B}_{B} \underline{1}_{E}$ and $f \underline{\underline{B}} \widetilde{1}_{E^{\prime}}$, where $\underline{1}_{E}$ denotes a fiber fundamental sequence induced by the identity $1_{E}: E \rightarrow E$. Such $f$ is called a fiber shape equivalence over $B$.

Proposition 1.7. If a fiber fundamental sequence $f=\left\{f_{n}, E, E^{\prime}\right\}_{Q, Q}$ over $B$ is a fiber shape equivalence over $B$, then for any compactum $B_{1} \subset B$ the restriction $f \mid p^{-1}\left(B_{1}\right)$ $=\left\{f_{n}, p^{-1}\left(B_{1}\right), p^{-1}\left(B_{1}\right)\right\}_{Q, Q}$ is a fiber shape equivalence over $B_{1}$.

A map $p: E \rightarrow B$ between compacta is shape shrinkable if $p$ induces a fiber shape equivalence from $p: E \rightarrow B$ to the identity $1_{B}: B \rightarrow B$. Let $p: S^{1} \times S^{1} \rightarrow S^{1}$ be the same as $[6$, p. 277 , Example]. Then it is easily seen that $p$ is fiber shape equivalent to the projection $q: p^{-1}(b) \times S^{1} \rightarrow S^{1}$ for $b \in S^{1}$, but $p$ is not fiber homotopy equivalent to the projection $q$.

\section{Fiber shape equivalences.}

In this section, we shall show that if a fiber fundamental sequence from a shape fibration to an approximate fibration is a shape equivalence, then it is a fiber shape equivalence. By using this result, we see that a map $p: E \rightarrow B$ between compact ANR's is shape shrinkable if and only if $p$ is a $C E$-map.

We need the following lemma.

Lemma 2.1. Let $E, E^{\prime}$ and $B$ be compacta and let $p: E \rightarrow B$ and $p^{\prime}: E^{\prime} \rightarrow B$ be shape fibrations. If a fiber fundamental sequence $f=\left\{f_{n}, E, E^{\prime}\right\}_{Q . Q}$ over $B$ is a weak domination (see [7, p. 8]) in shape category, then $f$ is a fiber weak domination, i.e., for any $\varepsilon>0$ and any neighborhood $U^{\prime}$ of $E^{\prime}$ in $Q$, there is a neighborhood $U$ of $E$ in $Q$ and an integer $n_{0}$ satisfying the conditions of Definition 1.1 such that for any $\eta>0$ and any neighborhood $W \subset U$ of $E$ in $Q$ there is a neighborhood $W^{\prime} \subset U^{\prime}$ of $E^{\prime}$ in $Q$, a map $g: W^{\prime} \rightarrow W$ and a homotopy $R: W^{\prime} \times I \rightarrow U^{\prime}$ such that

(1) $d\left(\tilde{p} g\left(e^{\prime}\right), \tilde{p}^{\prime}\left(e^{\prime}\right)\right)<\eta, \quad e^{\prime} \in W^{\prime}$,

(2) $R\left(e^{\prime}, 0\right)=e^{\prime}, \quad R\left(e^{\prime}, 1\right)=f_{n_{0}} g\left(e^{\prime}\right), \quad e^{\prime} \in W^{\prime}$ and

(3) $d\left(\tilde{p}^{\prime} R\left(e^{\prime}, t\right), \tilde{p}^{\prime}\left(e^{\prime}\right)\right)<\varepsilon, \quad e^{\prime} \in W^{\prime}, t \in I$.

Proof. Since $f=\left\{f_{n}, E, E^{\prime}\right\}_{Q, Q}$ is a fiber fundamental sequence, there is a neighborhood $U$ of $E$ in $Q$ and an integer $n_{0}$ such that for each $n \geq n_{0}$ there is a homotopy $F_{n_{0}, n}: U \times I \rightarrow U^{\prime}$ such that $F_{n_{0}, n}(e, 0)=f_{n_{0}}(e), F_{n_{0}, n}(e, 1)=f_{n}(e)$ and $d\left(\tilde{p}^{\prime} F_{n_{0}, n}(e, t), \tilde{p}(e)\right)<\varepsilon / 2$ for $e \in U, t \in I$. Let $\eta>0$ and $W$ be any neighborhood of $E$ in $Q$ with $W \subset U$. Since $p: E \rightarrow B$ and $p^{\prime}: E^{\prime} \rightarrow B$ are shape fibrations, by [11, 
Theorem 1], inductively we can find compact ANR's $E_{i}, E_{i}{ }^{\prime}, B_{i}(i=1,2,3)$ and $\varepsilon_{i}>$ $0, \delta_{i}>0(i=1,2)$ and an integer $n_{1} \geqq n_{0}$ such that

(1) $W \supset E_{1} \supset E_{2} \supset E_{3} \supset \operatorname{Int}_{Q} E_{3} \supset E, \quad U^{\prime} \supset E_{1}^{\prime} \supset E_{2}^{\prime} \supset E_{3}^{\prime} \supset \operatorname{Int} E_{Q} E_{3} \supset E^{\prime}$, $B_{1} \supset B_{2} \supset B_{3} \supset \operatorname{Int}_{Q} B_{3} \supset B$ and $\tilde{p}\left(E_{i}\right) \subset B_{i}, \tilde{p}^{\prime}\left(E_{i}^{\prime}\right) \subset B_{i} \quad(i=1,2,3)$,

(2) $\left(E_{2}{ }^{\prime}, \delta_{1}\right)$ is a lifting pair for $\left(E_{1}{ }^{\prime}, \varepsilon_{1}\right)$ and $\left(E_{3}, \delta_{2}\right)$ is a lifting pair for $\left(E_{2}, \varepsilon_{2}\right)$,

(3) any $2 \varepsilon_{2}$-near maps to $B_{2}$ are $\varepsilon_{1}$-homotopic and

(4) $\varepsilon_{1}<\varepsilon / 2, \quad \varepsilon_{2}<\operatorname{Min}\{\eta, \varepsilon / 2\}$ and $f_{n_{1}}\left(E_{i}\right) \subset E_{i}^{\prime} \quad(i=1,2,3)$, $d\left(\tilde{p}^{\prime} f_{n_{1}}\left|E_{3}, \tilde{p}\right| E_{3}\right)<\delta_{2}$ and $d\left(\tilde{p}^{\prime} f_{n_{1}}\left|E_{2}, \tilde{p}\right| E_{2}\right)<\varepsilon_{2}$.

Since $f$ is a weak domination in shape category, we may assume that there is a neighborhood $W^{\prime}$ of $E^{\prime}$ in $Q$ with $W^{\prime} \subset E_{3}{ }^{\prime}$, a map $g^{\prime}: W^{\prime} \rightarrow E_{3}$ and a homotopy $H: W^{\prime} \times I \rightarrow E_{3}{ }^{\prime}$ such that

(5) $H\left(e^{\prime}, 0\right)=e^{\prime}, \quad H\left(e^{\prime}, 1\right)=f_{n_{1}} g^{\prime}\left(e^{\prime}\right), \quad e^{\prime} \in W^{\prime}$.

By (4), d $\left(\tilde{p} g^{\prime}\left(e^{\prime}\right), \tilde{p}^{\prime} H\left(e^{\prime}, 1\right)\right)=d\left(\tilde{p} g^{\prime}\left(e^{\prime}\right), \tilde{p}^{\prime} f_{n_{1}} g^{\prime}\left(e^{\prime}\right)\right)<\delta_{2}, e^{\prime} \in W^{\prime} . \quad$ By (2) and [11, Proposition 1], there is a homotopy $\tilde{H}: W^{\prime} \times I \rightarrow E_{2}$ such that

(6) $\widetilde{H}\left(e^{\prime}, 1\right)=g^{\prime}\left(e^{\prime}\right), \quad e^{\prime} \in W^{\prime}$ and

(7) $d\left(\tilde{p} \tilde{H}, \tilde{p}^{\prime} H\right)<\varepsilon_{2}$.

Define a map $g: W^{\prime} \rightarrow E_{2} \subset W$ by

(8) $g\left(e^{\prime}\right)=\tilde{H}\left(e^{\prime}, 0\right), \quad e^{\prime} \in W^{\prime}$.

By (4), (5), (7) and (8) we have

(9) $d\left(\tilde{p} g\left(e^{\prime}\right), \tilde{p}^{\prime}\left(e^{\prime}\right)\right)<\varepsilon_{2}<\operatorname{Min}\{\eta, \varepsilon / 2\}$.

Define a homotopy $L: W^{\prime} \times[0,2] \rightarrow E_{2}{ }^{\prime}$ by

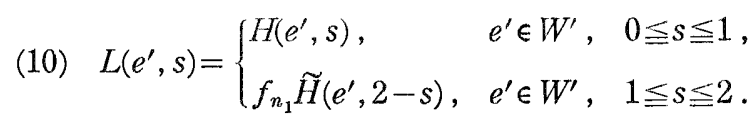

Then $L\left(e^{\prime}, 0\right)=e^{\prime}$ and $L\left(e^{\prime}, 2\right)=f_{n_{1}} g\left(e^{\prime}\right), e^{\prime} \in W^{\prime}$. By (4), (7) and (10),

(11) $d\left(\tilde{p}^{\prime} L\left(e^{\prime}, s\right), \tilde{p}^{\prime} L\left(e^{\prime}, 2-s\right)\right)=d\left(\tilde{p}^{\prime} H\left(e^{\prime}, s\right), \tilde{p}^{\prime} f_{n_{1}} \tilde{H}\left(e^{\prime}, s\right)\right)$

$\leqq d\left(\tilde{p}^{\prime} H\left(e^{\prime}, s\right), \tilde{p} \tilde{H}\left(e^{\prime}, s\right)\right)+d\left(\tilde{p} \tilde{H}\left(e^{\prime}, s\right), \tilde{p}^{\prime} f_{n_{1}} \widetilde{H}\left(e^{\prime}, s\right)\right)$

$<\varepsilon_{2}+\varepsilon_{2}=2 \varepsilon_{2}, \quad 0 \leqq s \leqq 1$.

By (3), there is a homotopy $K: W^{\prime} \times[0,2] \times[0,1\} \rightarrow B_{2}$ such that

(12) $K\left(e^{\prime}, s, t\right)=\tilde{p}^{\prime} L\left(e^{\prime}, s\right), \quad t \leqq 1-s$ or $t \leqq s-1$,

(13) $d\left(\tilde{p}^{\prime}\left(e^{\prime}\right), K\left(e^{\prime}, s, 1\right)\right)<\varepsilon_{1}, \quad 0 \leqq s \leqq 2$. 
Define a map $L^{\prime}: W^{\prime} \times(0 \times[0,1] \cup[0,2] \times 0 \cup 2 \times[0,1]) \rightarrow E_{2}^{\prime}$ by

(14) $L^{\prime}\left(e^{\prime}, s, t\right)= \begin{cases}L\left(e^{\prime}, 0\right), & s=0,0 \leqq t \leqq 1 \\ L\left(e^{\prime}, s\right), & 0 \leqq s \leqq 2, t=0, \\ L\left(e^{\prime}, 2\right), & s=2,0 \leqq t \leqq 1 .\end{cases}$

Then $\tilde{p}^{\prime} L^{\prime}=K \mid W^{\prime} \times(0 \times[0,1] \cup[0,2] \times 0 \cup 2 \times[0,1])$. By (2), there is a map $\tilde{K}: W^{\prime} \times$ $[0,2] \times[0,1] \rightarrow E_{1}^{\prime}$ such that

(15) $\tilde{K} \mid W^{\prime} \times(0 \times[0,1] \cup[0,2] \times 0 \cup 2 \times[0,1])=L^{\prime}$ and

(16) $d\left(\tilde{p}^{\prime} \tilde{K}, K\right)<\varepsilon_{1}$.

Define a homotopy $G: W^{\prime} \times[0,2] \rightarrow E_{\mathrm{i}}^{\prime}$ by

(17) $G\left(e^{\prime}, s\right)=\widetilde{K}\left(e^{\prime}, s, 1\right)$.

By (4),(13), (16) and (17) we have

(18) $d\left(\tilde{p}^{\prime} G\left(e^{\prime}, s\right), \tilde{p}^{\prime}\left(e^{\prime}\right)\right) \leqq d\left(\tilde{p}^{\prime} \widetilde{K}\left(e^{\prime}, s, 1\right), K\left(e^{\prime}, s, 1\right)\right)+d\left(K\left(e^{\prime}, s, 1\right), \tilde{p}^{\prime}\left(e^{\prime}\right)\right)$

$$
<\varepsilon_{1}+\varepsilon_{1}<\varepsilon .
$$

Then $G\left(e^{\prime}, 0\right)=e^{\prime}$ and $G\left(e^{\prime}, 2\right)=f_{n_{1}} g\left(e^{\prime}\right), e^{\prime} \in W^{\prime}$. Define a homotopy $R: W^{\prime} \times[0,3] \rightarrow U^{\prime}$ by

(19) $R\left(e^{\prime}, t\right)= \begin{cases}G\left(e^{\prime}, t\right), & 0 \leqq t \leqq 2, \\ F_{n_{0}, n_{1}}\left(g\left(e^{\prime}\right), 3-t\right), & 2 \leqq t \leqq 3 .\end{cases}$

Then $R\left(e^{\prime}, 0\right)=e^{\prime}, \quad R\left(e^{\prime}, 3\right)=f_{n_{0}} g\left(e^{\prime}\right)$ and $d\left(\tilde{p}^{\prime} R\left(e^{\prime}, t\right), \tilde{p}^{\prime}\left(e^{\prime}\right)\right)<\varepsilon$ for $e^{\prime} \in W^{\prime}, t \in[0,3]$. Hence $f$ is a fiber weak domination.

Corollary 2.2. Let $p: E \rightarrow B$ and $p^{\prime}: E^{\prime} \rightarrow B$ be shape fibrations between com pacta. If a fiber fundamental sequence $f=\left\{f_{n}, E, E^{\prime}\right\}_{Q, Q}$ is a weak domination in shape category, then for any compactum $B_{1} \subset B$, the restriction

$$
f \mid p^{-1}\left(B_{1}\right)=\left\{f_{n}, p^{-1}\left(B_{1}\right), p^{\prime-1}\left(B_{1}\right)\right\}_{Q, Q}
$$

is a fiber weak dominatian, hence we have the following.

(1) If $p^{-1}\left(B_{1}\right)$ is movable (see [3]), then $p^{\prime-1}\left(B_{1}\right)$ is movable.

(2) If $p^{-1}\left(B_{1}\right) \in A C^{n}$ (see [3]), then $p^{\prime-1}\left(B_{1}\right) \in A C^{n}$.

(3) If $p^{-1}\left(B_{1}\right)$ is an FAR (see [3]), then $p^{\prime-1}\left(B_{1}\right)$ is an FAR.

(4) $\mathrm{Fd}\left(p^{-1}\left(B_{1}\right)\right) \geqq \mathrm{Fd}\left(p^{\prime-1}\left(B_{1}\right)\right)$ (see $\left.[3]\right)$.

Theorem 2.3. Let $p: E \rightarrow B$ be a shape fibration from a compactum $E$ to $a$ compact $A N R$ B and let $p^{\prime}: E^{\prime} \rightarrow B$ be an approximate fibration between compact 
ANR's. Then a fiber fundamental sequence $f=\left\{f_{n}, E, E^{\prime}\right\}_{Q, Q}$ over $B$ is a fiber shape equivalence if and only if it is a shape equivalence.

Proof. It is enough to give the proof of sufficiency. Since $E^{\prime}$ and $B$ are ANR's, we may assume that there is a neighborhood $U$ of $E$ in $Q$ and an extension $\hat{p}: Q \rightarrow Q$ of $p: E \rightarrow B$ such that $f_{n}(U) \subset E^{\prime}$ for all $n$ and $\tilde{p}(U) \subset B$. Let $\varepsilon_{1}>\varepsilon_{2}>\varepsilon_{3}>\cdots$, be a sequence of positive numbers with $\lim \varepsilon_{i}=0$. Since $p$ is a shape fibration, inductively we can find a sequence $U \supset E_{1} \supset E_{1+1 / 2} \supset E_{2} \supset E_{2+1 / 2} \supset \cdots$, of compact ANR's, an increasing sequence $k_{1}<k_{2}<k_{3}<\cdots$, of natural numbers and a sequence $\delta_{1}>\delta_{2}>$ $\delta_{3}>\cdots,\left(\delta_{i}<\varepsilon_{i}\right)$ of positive numbers such that

(1) Int $E_{Q} \supset E$ and $\bigcap_{i=1}^{\infty} E_{i}=E$,

(2) $\left(E_{i+1 / 2}, 2 \delta_{i}\right)$ is a lifting pair for $\left(E_{i}, \varepsilon_{i} / 2\right), i=1,2, \cdots$, and

(3) for each $k \geqq k_{i}$, there is a homotopy $F_{k_{i, k}}: E_{i} \times I \rightarrow E^{\prime}$ such that $F_{k_{i}, k}(e, 0)=$ $f_{k_{i}}(e), F_{k_{i}, k}(e, 1)=f_{k}(e)$ and $d\left(p^{\prime} F_{k_{i}, k}(e, t), \tilde{p}((e))<\varepsilon_{i}, e \in E_{i}, t \in I\right.$.

Since $p^{\prime}: E^{\prime} \rightarrow B$ is an approximate fibration, there is a sequence $\delta_{1}{ }^{\prime}>\delta_{2}{ }^{\prime}>\delta_{3}{ }^{\prime}>\cdots$, $\left(\delta_{i}{ }^{\prime}<\delta_{i}\right)$ of positive numbers such that $\left(E^{\prime}, \delta_{i}{ }^{\prime}\right)$ is a lifting pair for $\left(E^{\prime}, \delta_{i}\right)$. By Lemma 2.1 , we may assume that there is a map $g_{i}: E^{\prime} \rightarrow E_{i+1 / 2}$ and a homotopy $R_{i}: E^{\prime} \times I \rightarrow$ $E^{\prime}$ for each $i$ such that

(4) $d\left(\tilde{p} g_{i}\left(e^{\prime}\right), p^{\prime}\left(e^{\prime}\right)\right)<\delta_{i}{ }^{\prime}, \quad e^{\prime} \in E^{\prime}$,

(5) $R_{i}\left(e^{\prime}, 0\right)=e^{\prime}, \quad R_{i}\left(e^{\prime}, 1\right)=f_{k_{i}} g_{i}\left(e^{\prime}\right), \quad e^{\prime} \in E^{\prime} \quad$ and

(6) $d\left(p^{\prime} R_{i}\left(e^{\prime}, t\right), p^{\prime}\left(e^{\prime}\right)\right)<\varepsilon_{i}, \quad e^{\prime} \in E^{\prime}, t \in I$.

Since $f$ is a shape equivalence, by the construction of $g_{i}$ (see the proof of Lemma 2.1) we may assume that there is a homotopy $L_{i}: E_{i+1} \times I \rightarrow E_{i+1 / 2}$ with $L_{i}(e, 0)=e$, $L_{i}(e, 1)=g_{i} f_{k_{i+1}}(e), e \in E_{i+1}$. By (4), we have

(7) $d\left(\tilde{p} L_{i}(e, 1), p^{\prime} f_{k_{i+1}}(e)\right)=d\left(\tilde{p} g_{i} f_{k_{i+1}}(e), p^{\prime} f_{k_{i+1}}(e)\right)<\delta_{i}{ }^{\prime}, \quad e \in E_{i+1}$.

Hence, by (2) and the same way as the proof of Lemma 2.1, there is a map $f_{k i+1}^{\prime}: E_{i+1} \rightarrow E^{\prime}$ and a homotopy $M_{i}: E_{i+1} \times I \rightarrow E_{i}$ such that

(8) $\mathrm{d}\left(p^{\prime} f_{k_{i+1}}^{\prime}(e), \tilde{p}(e)\right)<\delta_{i}, \quad e \in E_{i+1}$,

(9) $M_{i}(e, 0)=e, \quad M_{i}(e, 1)=g_{i} f_{k_{i+1}}^{\prime}(e), e \in E_{i+1}$ and

(10) $d\left(\tilde{p} M_{i}(e, t), \hat{p}(e)\right)<\varepsilon_{i}, \quad e \in E_{i+1}$.

By (3), (5) and (9), we can define a homotopy $G_{i}: E_{i+1} \times[0,3] \rightarrow E^{\prime}$ by 
(11) $\quad G_{i}(e, t)= \begin{cases}R_{i}\left(f_{k_{i+1}}^{\prime}(e), t\right), & 0 \leqq t \leqq 1 \\ f_{k_{i}} M_{i}(e, 2-t), & 1 \leqq t \leqq 2, \\ F_{k_{i}, k_{i+1}}(e, t-2), & 2 \leqq t \leqq 3 .\end{cases}$

By (3), (6), (10) and (11), we have

(12) $G_{i}(e, 0)=f_{k_{i+1}}^{\prime}(e), \quad G_{i}(e, 3)=f_{k_{i+1}}(e), \quad e \in E_{i+1} \quad$ and

(13) $d\left(p^{\prime} G_{i}(e, t), \tilde{p}(e)\right)<2 \varepsilon_{i}, \quad e \in E_{i+1}, \quad 0 \leqq t \leqq 3$.

By (12) and (13), we obtain a fiber fundamental sequence $f^{\prime}$ over $B$ induced by $\left\{f_{k i}^{\prime}: E_{k_{i} \rightarrow E^{\prime}}\right\}$ such that $f^{\prime} \widetilde{\sim} f$. By (5), (9) and (12), we can define a homotopy $S_{i}: E^{\prime} \times[0,5] \rightarrow E_{i}$ by

(14) $S_{i}\left(e^{\prime}, t\right)= \begin{cases}M_{i}\left(g_{i+1}\left(e^{\prime}\right), t\right), & 0 \leqq t \leqq 1, \\ g_{i} G_{i}\left(g_{i+1}\left(e^{\prime}\right), t-1\right), & 1 \leqq t \leqq 4 \\ g_{i} R_{i+1}\left(e^{\prime}, 5-t\right), & 4 \leqq t \leqq 5 .\end{cases}$

Then $S_{i}\left(e^{\prime}, 0\right)=g_{i+1}\left(e^{\prime}\right), S_{i}\left(e^{\prime}, 5\right)=g_{i}\left(e^{\prime}\right), e^{\prime} \in E^{\prime}$. Also by (4), (6), (10), (13) and (14), we have $d\left(\tilde{p} S_{i}\left(e^{\prime}, t\right), p^{\prime}\left(e^{\prime}\right)\right)<4 \varepsilon_{i}$ for $e^{\prime} \in E^{\prime} \quad 0 \leqq t \leqq 5$. Hence we obtain a fiber fundamental sequence $g$ over $B$ induced by $\left\{g_{i}: E^{\prime} \rightarrow E_{i}\right\}$. By (9) and (10), we conclude that

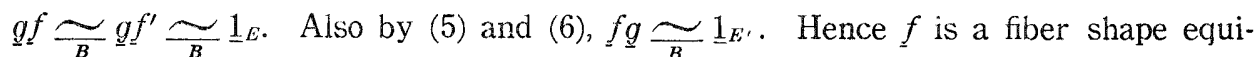
valence over $B$.

Corollary 2.4. Let $p: E \rightarrow B$ and $p^{\prime}: E^{\prime} \rightarrow B$ be approximate fibrations between compact ANR's. If a fiber fundamental sequence $f=\left\{f_{n} E, E^{\prime}\right\}_{Q, Q}$ over $B$ is a shape equivalence, then it is a fiber shape equivalence. In particular, if a fiber map $f: E \rightarrow$ $E^{\prime}$ over $B$ is a homotopy equivalence, it is a fiber shape equivalence.

The next result follows from Vietoris-Smale theorem, [10, Lemma 2.3 or 11, Theorem 4], Corollary 1.7 and 2.4 .

Corollary 2.5. Let $p: E \rightarrow B$ be a map between compact ANR's. Then the following are equivalent.

(1) $p$ is a CE-map.

(2) $p$ is a homotopy equivalence and an approximate fibration.

(3) $p$ is shape shrinkable.

(4) $p$ is a hereditary shape equivalence.

\section{The Complement Theorem of fiber shape equivalences.}

In this section, we prove the following theorem. 
Theorem 3.1. Let $E, E^{\prime}$ and $B$ be compacta in the Hilbert cube $Q$ and let $E, E^{\prime} \subset Q$ be Z-sets. Then a map $p: E \rightarrow B$ over $B$ is fiber shape equivalent to a map $p^{\prime}: E^{\prime} \rightarrow B$ over $B$ if and only if there is a homeomorphism $h: Q-E \cong Q-E^{\prime}$ such that for each $b \in B$ and each neighborhood $W^{\prime}$ of $p^{-1}(b)$ in $Q$, there is a neighborhood $W$ of $p^{-1}(b)$ in $Q$ such that $h(W-E) \subset W^{\prime}-E^{\prime}$.

Corollary 3.2. Let $E, E^{\prime}$ and $B$ be compacta in the Hilbert cube $Q$ and let $E, E^{\prime} \subset Q$ be $Z$-sets. Then a map $p: E \rightarrow B$ over $B$ is fiber shape equivalent to a map $p^{\prime}: E^{\prime} \rightarrow B$ over $B$ if and only if there is a homeomorphism $h: Q-E \cong Q-E^{\prime}$ such that for any extension $\tilde{p}^{\prime}: Q \rightarrow Q$ of $p^{\prime}$ there is the extension $\tilde{p}: Q \rightarrow Q$ of $p$ such that $\tilde{p} \mid Q-E=\tilde{p}^{\prime} h$.

Corollary 3.3. Let $E$ and $B$ be $Z$-sets in the Hilbert cube $Q$. Then a map $p: E \rightarrow B$ is shape shrinkable if and only if there is an extension $\tilde{p}: Q \rightarrow Q$ of $p$ such that $\tilde{p} \mid Q-E: Q-E \cong Q-B$ is a homeomorphism.

Let $U$ be a collection of subsets of a space $Y$. A map $f: X \rightarrow Y$ is $U$-close to a map $g: X \rightarrow Y$ if for each $x \in X$, there is $U \in \mathcal{U}$ such that $\{f(x), g(x)\} \subset U$. A homotopy $H: X \times I \rightarrow Y$ is $q$-limited if for each $x \in X$ there is $U \in \mathcal{U}$ such that $H(\{x\} \times$ $I) \subset U$. A closed subset $A$ in a space $X$ is a $Z$-set in $X$ if for each open cover $U$ of $X$ there is a map of $X$ into $X-A$ which is $U$-close to the identity $1_{X}$. A map $f: A \rightarrow X$ is a $Z$-embedding if $f$ is an embedding and $f(A)$ is a $Z$-set in $X$.

The proof of Theorem 3.1 is analogous to one of Chapman's [4], but much sharper results will be used. We need the followings.

Lemma 3.4 (see [1, Theorem 3.1] or [5, Theorem 11.2]). If $\left(A, A_{0}\right)$ is a compact pair and $f: A \rightarrow Q$ is a map such that $f \mid A_{0}$ is a Z-embedding, then for any open cover $U$ of $Q$ there is a $Z$-embedding $g: A \rightarrow Q$ such that $g\left|A_{0}=f\right| A_{0}$ and $g$ is $U$ close to $f$.

LEMma 3.5 (see [1, Theorem 6.1] or [5, Theorem 19.4]). Let $M$ be a Q-manifold, $A$ be a compactum and let $F: A \times I \rightarrow M$ be a map such that $F_{0}$ and $F_{1}$ are $Z$ embeddings. If $F$ is $U$-limited for an open cover $U$ of $M$, then there is an isotopy $H: M \times I \rightarrow M$ such that $H_{0}=i d, H_{1} F_{0}=F_{1}$ and $H$ is $U$-limited.

Proof of THEOREM 3.1. Let $h: Q-E \cong Q-E^{\prime}$ be a homeomorphism satisfying

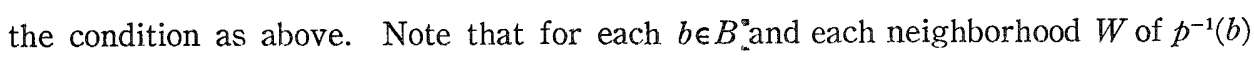
in $Q$ there is a neighborhood $W^{\prime}$ of $p^{\prime-1}(b)$ in $Q$ such that $h^{-1}\left(W^{\prime}-E^{\prime}\right) \subset W-E$. In fact, suppose, on the contrary, that there is a sequence $\left\{x_{i}{ }^{\prime}\right\}_{i=1,2, \ldots}$ such that $x_{i}{ }^{\prime} \epsilon$ 
$Q-E^{\prime}, \lim _{i \rightarrow \infty} x_{i}{ }^{\prime}=x^{\prime} \in p^{\prime-1}(b)$ and $h^{-1}\left(x_{i}{ }^{\prime}\right) \in Q-W$ for each $i$. Choose a subsequence $\left\{x_{n_{i}}^{\prime}\right\}$ of $\left\{x_{i}{ }^{\prime}\right\}$ such that $\lim _{i \rightarrow \infty} h^{-1}\left(x_{n_{i}}^{\prime}\right)=x \in E-p(b)$. Let $W_{1}^{\prime}$ and $W_{2}^{\prime}$ be neighborhoods of $p^{\prime-1}(b)$ and $p^{\prime-1}(p(x))$ in $Q$, respectively, such that $W_{1}^{\prime} \cap W_{2}^{\prime}=\phi$. Since there is a neighborhood $W_{2}$ of $p^{-1}(p(x))$ in $Q$ such that $h\left(W_{2}-E\right) \subset W_{2}^{\prime}-E^{\prime}$ and $h^{-1}\left(x_{n_{i}}^{\prime}\right) \in W_{2}$ for almost all $i, h\left(h^{-1}\left(x_{n_{i}}^{\prime}\right)\right)=x_{n_{i}}^{\prime} \in W_{2}^{\prime}$, which implies the contradiction.

Since $E \subset Q$ is a $Z$-set, there is a homotopy $F: Q \times I \rightarrow Q$ such that $F(q, 0)=q$, $F(q, t) \in Q-E$, for $q \in Q, 0<t \leqq 1$. Similarly there is a homotopy $G: Q \times I \rightarrow Q$ such that $G(q, 0)=q, G(q, t) \in Q-E^{\prime}$, for $q \in Q, 0<t \leqq 1$. Define maps $f_{n}: Q \rightarrow Q$ and $g_{n}: Q \rightarrow$ $Q$ for each integer $n$ by $f_{n}(q)=h(F(q, 1 / n)), g_{n}(q)=h^{-1}(G(q, 1 / n))$, for $q \in Q$. Consider $\underline{f}=\left\{f_{n}, E, E^{\prime}\right\}_{Q, Q}$ and $\underline{g}=\left\{g_{n}, E^{\prime}, E\right\}_{Q, Q}$. Then we shall show that $\underline{f}$ and $\underline{g}$ are fiber fundamental sequences over $B$ such that $g f \widetilde{\sim}_{B} \underline{1}_{E}$ and $g f \widetilde{B}_{B} \underline{1}_{E^{\prime}}$. Let $\hat{p}: Q \rightarrow Q$ and $\tilde{p}^{\prime}: Q \rightarrow Q$ be extensions of $p$ and $p^{\prime}$, respectively. Let $\stackrel{B}{U^{\prime}}$ be a neighborhood of $E^{\prime}$ in $Q$ and let $\varepsilon>0$. For each $b \in B$, choose a neighborhood $C_{b}$ of $b$ in $Q$ such that diam $C_{b}<\varepsilon / 2$. Then there is a neighborhood $W_{b}$ of $p^{-1}(b)$ in $Q$ such that $h\left(W_{b}-E\right) \subset\left[U^{\prime} \cap \tilde{p}_{m}^{\prime-1}\left(C_{b}\right)\right]-E^{\prime}$ and $\tilde{p}\left(W_{b}\right) \subset C_{b}$. Choose a finite collection $\left\{W_{b_{1}}, W_{b_{2}}, \cdots\right.$, $\left.W_{b_{m}}\right\}$ such that $\bigcup_{i=1}^{m} W_{b_{i}} \supset E$. Also choose a neighborhood $U$ of $E$ in $Q$ and an integer

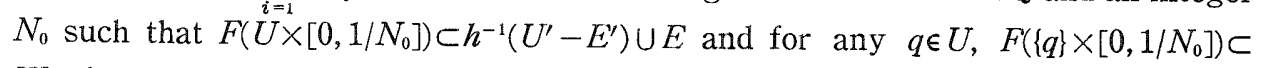
$W_{b_{i}}$ for some $i$. For each $n \geqq N_{0}$, define a homotopy $H: U \times[1 / n+1,1 / n] \rightarrow U^{\prime}$ by $H(q, t)=h(F(q, t))$, for $q \in U, 1 / n+1 \leqq l \leqq 1 / n$. Then $H(q, 1 / n+1)=f_{n+1}(q), H(q, 1 / n)=f_{n}(q)$ and $d\left(\tilde{p}(q), \tilde{p}^{\prime} H(q, t)\right)<\varepsilon$, for $q \in U, 1 / n+1 \leqq t \leqq 1 / n$. Hence $f$ is a fiber fundamental sequence over $B$. Similarly, $g$ is a fiber fundamental sequence over $B$. To see that $g f \underset{B}{\widetilde{1}} \underline{1}_{E}$, choose a neighborhood $U$ of $E$ in $Q$ and $\varepsilon>0$. By the same way as above, we can choose a neighborhood $V^{\prime}$ of $E^{\prime}$ in $Q$ and $\varepsilon_{1}>0$ such that $h^{-1}\left(V^{\prime}-\right.$ $\left.E^{\prime}\right) \subset U-E$ and $h^{-1} G(q, t) \in U$, for $q \in V^{\prime}, 0<t \leqq \varepsilon_{1}$ and $d\left(\tilde{p} h^{-1} G(q, t), \tilde{p}^{\prime}(q)\right)<\varepsilon / 2$, for $q \in V^{\prime}$, $0<t \leqq \varepsilon_{1}$. Choose a small neighborhood $V$ of $E$ in $Q(V \subset U)$ and $\varepsilon_{2}>0\left(\varepsilon_{2}<\varepsilon_{1}\right)$ such that $h F(q, t) \in V^{\prime}$, for $q \in V, 0<t \leqq \varepsilon_{2}$ and $d\left(\tilde{p}^{\prime} h F(q, t), \tilde{p}(q)\right)<\varepsilon / 2$, for $q \in V, 0<t \leqq \varepsilon_{2}$. Also, we may assume that $d(\tilde{p} F(q, t), \tilde{p}(q))<\varepsilon / 2$, for $q \in V, 0 \leqq t \leqq \varepsilon_{2}$. Let $N_{1}$ be an integer sucn that $\varepsilon_{2}>1 / N_{1}$. Then for each $n \geqq N_{1}$, we can define a homotopy $H: V \times I \rightarrow U$ by

$$
H(q, t)= \begin{cases}h^{-1} G(h F(q, 1 / n), 1 / n-t), & q \in V, 0 \leqq t \leqq 1 / n, \\ F(q,(t-1) /(1-n)), & q \in V, 1 / n \leqq t \leqq 1 .\end{cases}
$$

Then $H(q, 0)=g_{n} f_{n}(q), H(q, 1)=q$ for $q \in V d(\tilde{p}(q), \tilde{p} H(q, t))<\varepsilon$, for $q \in V, t \in I$. Hence, $g f \widetilde{\boldsymbol{B}} \underline{1}_{E}$. Similarly, $f \underline{\underline{B}} \widetilde{\boldsymbol{B}}_{E^{\prime}}$. Thus $p$ is fiber shape equivalent to $p^{\prime}$ over $B$.

Conversely, we shall construct a homeomorphism $h: Q-E \cong Q-E^{\prime}$ satisfying the condition of Theorem 3.1. Let $\tilde{p}: Q \rightarrow Q$ and $\tilde{p}^{\prime}: Q \rightarrow Q$ be extensions of $p$ and $p^{\prime}$, respectively. Let $f=\left\{f_{n}, E, E^{\prime}\right\}_{Q, Q}$ and $\underline{g}=\left\{g_{n}, E^{\prime}, E\right\}_{Q, Q}$ be fiber fundamental 


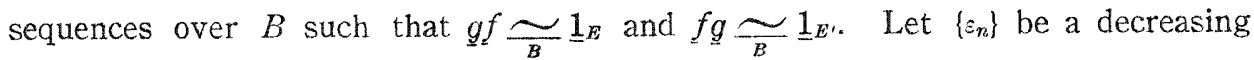
sequence of positive numbers such that $\sum_{n=1}^{\infty} \varepsilon_{n}<\infty$. We inductively construct sequences $\left\{U_{n}\right\}$ and $\left\{V_{n}\right\}$ of open sets in $Q$ and a sequence $\left\{h_{n}\right\}$ of homeomorphisms of $Q$ onto itself satisfying the following properties.

(1) $E=\bigcap_{n=1}^{\infty} U_{n}$ and $U_{n+1} \subset U_{n}$, for each $n \geqslant 1$,

(2) $E^{\prime}=\bigcap_{n=1}^{\infty} V_{n}$ and $V_{n+1} \subset V_{n}$, for each $n \geqq 1$,

(3) $h_{i} \cdots h_{2} h_{1}\left(U_{n}\right) \subset V_{n}$, for $i \geqq 2 n-1$,

(4) $h_{i} \cdots h_{2} h_{1}\left(U_{n}\right) \supset V_{n+1}$, for $i \geqq 2 n$,

(5) $h_{i} \mid Q-V_{n}=i d$, for $i \geqq 2 n$,

(6) $h_{i} \mid Q-h_{2 n} \cdots h_{2} h_{1}\left(U_{n}\right)=i d$, for $i \geqq 2 n+1$,

(7) $d\left(\tilde{p}^{\prime} h_{i}(q), \tilde{p}^{\prime}(q)\right)<4 \varepsilon_{n}$, for $q \in Q, i \geqq 2 n$,

(8) $d\left(\tilde{p}^{\prime} h_{i} \cdots h_{2} h_{1}(z), b\right)<2 \varepsilon_{n}$, for $b \in B, z \in p^{-1}(b)$ and $i \geqq 2 n-1$.

First, we will construct a homeomorphism $h_{1}: Q \rightarrow Q$. Let $V$, be a small neighborhood of $E^{\prime}$ in $Q$. Since $f$ is a fiber fundamental sequence over $B$, there is an integer $N_{1}$ and a neighborhood $E_{1}$ of $E$ in $Q$ such that for $m \geqq n \geqq N_{1}$ there is a homotopy $F_{n, m}: E_{1} \times I \rightarrow V_{1}$ such that $F_{n, m}(q, 0)=f_{n}(q), \quad F_{n, m}(q, 1)=f_{m}(q)$ and $d\left(\tilde{p}^{\prime} F_{n, m}(q, t), \tilde{p}(q)\right)<\varepsilon_{1} / 2$, for $q \in E_{1}, t \in I$. By Lemma 3.4, there is a $Z$-embedding $\alpha_{\mathrm{t}}: E$ $\rightarrow V_{1}$ such that there is a homotopy $H: E \times I \rightarrow V_{1}$ such that $H_{0}=\alpha_{1}$ and $H_{1}=f_{N_{1}} \mid E$ and $d\left(\tilde{p}^{\prime} H(q, t), \tilde{p}(q)\right)<\varepsilon_{1} / 2$, for $q \in E, t \in I$. By Lemma 3.5, there is a homeomorphism $h_{1}: Q \rightarrow Q$ such that $h_{1} \mid E=\alpha_{1}$. Since $V_{1}$ is an ANR, we may assume that there is an extension $\widetilde{H}: E_{1} \times I \rightarrow V_{1}$ of $H$ such that $\widetilde{H}_{0}=h_{1}\left|E_{1}, \widetilde{H}_{1}=f_{N_{1}}\right| E_{1}$ and $d\left(\tilde{p}^{\prime} \tilde{H}(q, t), \tilde{p}(q)\right)$ $<\varepsilon_{1} / 2$, for $q \in E_{1}, t \in I$.

Next, we will construct a homeomorphism $h_{2}: Q \rightarrow Q$. Let $U_{1} \subset E_{1}$ be a small neighborhood of $E$ in $Q$ such that $h_{1}\left(U_{1}\right) \subset V_{1}$. Since $g$ is a fiber fundamental sequence over $B$, there is an integer $N_{2} \geqq N_{1}$ and a neighborhood $E_{1}{ }^{\prime}$ of $E^{\prime}$ in $Q$ such that for $m \geqq n \geqq N_{2}$ there is a homotopy $G_{n, m}: E_{1}{ }^{\prime} \times I \rightarrow U_{1}$ such that $G_{n, m}(q, 0)=$ $g_{n}(q), G_{n, m}(q, 1)=g_{m}(q)$ and $d\left(\tilde{p} G_{n, m}(q, t), \tilde{p}^{\prime}(q)\right)<\varepsilon_{1} / 2$, for $q \in E_{1}{ }^{\prime}, t \in I$. By Lemma 3.4, there is a $Z$-embeding $\alpha_{2}: E^{\prime} \rightarrow U_{1}$ and a homotopy $K: E^{\prime} \times I \rightarrow U_{1}$ such that $K_{0}=\alpha_{2}$, $K_{1}=g_{N_{2}} \mid E^{\prime}$ and $d\left(\tilde{p} K(q, t), \tilde{p}^{\prime}(q)\right)<\varepsilon_{1} / 2$, for $q \in E^{\prime}, t \in I$. By choosing $N_{2}$ sufficiently large, we may assume that there is a homotopy $L: E^{\prime} \times I \rightarrow V_{1}$ such that $L_{0}=f_{N_{2}} g_{N_{2}} \mid E^{\prime}$, $L_{1}=i d$ and $d\left(\tilde{p}^{\prime} L(q, t), \tilde{p}^{\prime}(q)\right)<\varepsilon_{1}$, for $q \in E^{\prime}, t \in I$. Define a homotopy $M: E^{\prime} \times I \rightarrow V_{1}$ by 


$$
M(q, t)= \begin{cases}h_{1} K(q, 4 t), & q \in E^{\prime}, 0 \leqq t \leqq 1 / 4, \\ \widetilde{H}\left(g_{N_{2}}(q), 4 t-1\right), & q \in E^{\prime}, 1 / 4 \leqq t \leqq 1 / 2, \\ F_{N_{1}, N_{2}}\left(g_{N_{2}}(q), 4 t-2\right), & q \in E^{\prime}, 1 / 2 \leqq t \leqq 3 / 4, \\ L(q, 4 t-3), & q \in E^{\prime}, 3 / 4 \leqq t \leqq 1 .\end{cases}
$$

Then $M_{0}=h_{1} \alpha_{2}, M_{1}=i d$ and $d\left(\tilde{p}^{\prime} M(q, t), \tilde{p}^{\prime}(q)\right)<\varepsilon_{1}$, for $q \in E^{\prime}, t \in I$. By Lemma 3.5, we may assume that there is a homeomorphism $h_{2}{ }^{\prime}: Q \rightarrow Q$ such that $h_{2}{ }^{\prime} \mid E^{\prime}=h_{1} \alpha_{2}$, $h_{2}{ }^{\prime} \mid Q-V_{1}=i d$ and $d\left(\tilde{p}^{\prime} h_{2}{ }^{\prime}(q), \tilde{p}^{\prime}(q)\right)<\varepsilon_{1}$, for $q \in Q$. Let $h_{2}=\left(h_{2}{ }^{\prime}\right)^{-1}$.

Also, we will construct a homeomorphism $h_{3}: Q \rightarrow Q$. Since $h_{1}\left(U_{1}\right)$ is an ANR, there is a neighborhood $E_{2}{ }^{\prime}\left(E_{2}{ }^{\prime} \subset E_{1}{ }^{\prime}\right)$ of $E^{\prime}$ in $Q$ such that $E^{\prime} \subset E_{2}{ }^{\prime} \subset h_{2} h_{1}\left(U_{1}\right)$ and there is an extension $\tilde{K}: E_{2}{ }^{\prime} \times I \rightarrow h_{1}\left(U_{1}\right)$ of $h_{1} K$ such that $\widetilde{K}_{0}=h_{2}{ }^{\prime}\left|E_{2}{ }^{\prime}, \widetilde{K}_{1}=h_{1} g_{N_{2}}\right| E_{2}{ }^{\prime}$ and $d\left(\tilde{p}^{\prime} \tilde{K}(q, t), \tilde{p}^{\prime}(q)\right)<\varepsilon_{1}$, for $q \in E_{2}{ }^{\prime}, t \in I$. Let $V_{2}$ be a small neighborhood of $E^{\prime}$ in $Q$ such that $V_{2} \subset V_{1}, V_{2} \subset E_{2}^{\prime}$. Since $\underline{f}$ is a fiber fundamental sequence over $B$, there is an integer $N_{3} \geqq N_{2}$ and a neighborhood $E_{2} \subset E_{1}$ of $E$ in $Q$ such that for $m \geqq n \geqq N_{3}$ there is a homotopy $F_{n, m}: E_{2} \times I \rightarrow V_{2}$ such that $F_{n, m}(q, 0)=f_{n}(q), F_{n, m}(q, 1)$ $=f_{m}(q)$ and $d\left(\tilde{p}^{\prime} F_{n, m}(q, t), \tilde{p}(q)\right)<\varepsilon_{2} / 2$, for $q \in E_{2}, t \in I$. Choose a $Z$-embedding $\alpha_{3}: E \rightarrow$ $V_{2}$ and a homotopy $R: E \times I \rightarrow V_{2}$ such that $R_{0}=\alpha_{3}, R_{1}=f_{N_{3}} \mid E$ and $d\left(\tilde{p}^{\prime} R(q, t), \tilde{p}(q)\right)<$ $\varepsilon_{2} / 2$, for $q \in E, t \in I$. By choosing $N_{3}$ large, there is a homotopy $D: E \times I \rightarrow U_{1}$ such that $D_{0}=g_{N_{3}} f_{N_{3}} \mid E, D_{1}=i d$ and $d(\tilde{p} D(q, t), \tilde{p}(q))<\varepsilon_{1}$, for $q \in E, t \in I$. Then we can define a homotopy $T: E \times I \rightarrow h_{2} h_{1}\left(U_{1}\right)$ by

$$
T(q, t)= \begin{cases}R(q, 4 t), & q \in E, 0 \leqq t \leqq 1 / 4, \\ h_{2} \tilde{K}\left(f_{N_{3}}(q), 4 t-1\right), & q \in E, 1 / 4 \leqq t \leqq 1 / 2, \\ h_{2} h_{1} G_{N_{2}, N_{3}}\left(f_{N_{3}}(q), 4 t-2\right), & q \in E, 1 / 2 \leqq t \leqq 3 / 4 \\ h_{2} h_{1} D(q, 4 t-3), & q \in E, 3 / 4 \leqq t \leqq 1\end{cases}
$$

Then $T_{0}=\alpha_{3}, T_{1}=h_{2} h_{1} \mid E$ and $d\left(\tilde{p}^{\prime} T(q, t), \tilde{p}(q)\right)<4 \varepsilon_{1}$, for $q \in E, t \in I$. By Lemma 3.5, there is a homeomorphism $h_{3}: Q \rightarrow Q$ such that $h_{3}\left|Q-h_{2} h_{1}\left(U_{\imath}\right)=i d, h_{3} h_{2} h_{1}\right| E=\alpha_{3}$ and $d\left(\tilde{p}^{\prime} h_{3}(q), \tilde{p}^{\prime}(q)\right)<4 \varepsilon_{1}$, for $q \in Q$.

If we continue the process as above, we have desired sequences $\left\{U_{n}\right\},\left\{V_{n}\right\}$ and $\left\{h_{n}\right\}$ satisfying the properties $(1)-(8)$ as we wanted. Define a map $h: Q-E \rightarrow Q-E^{\prime}$ by $h(q)=\lim _{j \rightarrow \infty} h_{j} \cdots h_{2} h_{1}(q)$ for $q \in Q-E$. By (1)-(6), $h$ is a homeomorphism (see [4]). To prove that $h$ is a desired homeomorphism, for each $b \in B$ choose a neighborhood $W^{\prime}$ of $p^{\prime-1}(b)$ in $Q$. Let $N_{0}$ be an integer and $\varepsilon>0$ such that $V_{N_{0}} \cap \tilde{p}^{\prime-1}(B(b ; \varepsilon)) \subset$ $W^{\prime}$, where $B(b ; \varepsilon)=\{x \in Q \mid d(x, b)<\varepsilon\}$. Choose an integer $n_{0}$ such that $\sum_{n=n_{0}}^{\infty} 4 \varepsilon_{n}<\varepsilon / 2$. By (8), $h_{i} \cdots h_{2} h_{1}\left(p^{-1}(b)\right) \subset \tilde{p}^{\prime-1}(B(b ; \varepsilon / 2))$ for $i \geqq 2 n_{0}-1$. Let $n_{3}=\operatorname{Max}\left\{N_{0}, n_{0}\right\}$. Choose a neighborhood $W$ of $p^{-1}(b)$ in $Q$ such that $W \subset U_{n_{1}}$ and $h_{2 n_{1}-1} \cdots h_{2} h_{1}(W) \subset \tilde{p}^{\prime-1}(B(b$; $\varepsilon / 2))$. By (3) and (7), $h_{i} h_{i-1} \cdots h_{2} h_{1}(W) \subset V_{N_{0}} \cap \tilde{p}^{\prime-1}(B(b ; \varepsilon))$ for $i \geqq 2 n_{1}-1$. Hence $h(W-$ 
$E) \subset W^{\prime}-E^{\prime}$. This completes the proof.

Corollary 3.4. Let $p: E \rightarrow B$ be a CE-map from a compact ANR $E$ to a compactum $B$. Then $p$ is shape shrinkable if and only if $B$ is an ANR.

Proof. Sufficiency follows from Corollory 2.5. Suppose that $p$ is shape shrinkable. By Corollary 3.3, there is an extension $\tilde{p}: Q \rightarrow Q$ of $p$ such that $\tilde{p} \mid Q-E: Q-$ $E \cong Q-B$ is a homeomorphism. Since $E$ is an $A N R$, there is a neighborhood $U$ of $E$ in $Q$ and a retraction $r: U \rightarrow E$. Clearly, there is a retraction $r^{\prime}: \tilde{p}(U) \rightarrow B$ such that $\tilde{p} r(x)=r^{\prime} \tilde{p}(x)$ for $x \in U$. Hence $B$ is an ANR.

In [9], Kozłowski proved the following. If $E$ and $B$ are $Z$-sets in the Hilbert cube $\mathrm{Q}$, then a map $p: E \rightarrow B$ between compacta is a hereditary shape equivalence iff there is an extension $\tilde{p}: Q \rightarrow Q$ of $p$ such that $\tilde{p} \mid Q-E: Q-E \cong Q-B$ is a homeomorphism. Hence by Corollary 3.3 we have the following.

Corollary 3.5. Let $p: E \rightarrow B$ be a map between compacta. Then $p$ is shape shrinkable if and only if $p$ is a hereditary shape equivalence.

By Theorem 3.1 and Corollary 3.5, we can easily see the following.

COROLlaRY 3.6. Fiber shape equivalences preserve shape fibrations. In particular, hereditary shape equivalences are shape fibrations.

Finally, the author would like to thank the referee for helpful comments.

\section{References}

[1] Anderson, R.D. and Chapman, T.A., Extending homeomorphisms to Hilbert cube manifolds, Pacific J. Math., 38 (1971) pp. 281-293.

[2] Borsuk, K., Concerning homotopy properties of compacta, Fund. Math., 62 (1968) pp. 223-254.

[3] - Theory of shape, PWN. Warszawa 1975.

[4] Chapman, T.A., On some applications of infinite-dimensional manifolds to the theory of shape, Fund. Math., 76 (1972) pp. 181-193.

[5] Lectures of Hilbert cube manifolds, CBMS Regional Conference Series in Mathematics, Amer. Math. Soc., Providence 1976.

[6] Coram, D. and Duvall, P., Approximate fibrations, Rocky Mountain J. Math., 7 (1977) pp. 275-288.

[7] Dydak, J., The Whitehead and Smale theorems in shape theory, Dissertationes Math., Warszawa 1979.

[8] Jani, M., Induced shape fibrations and fiber shape equivalences, $\mathrm{Ph}$. D. dissertation (1978), the City University of New York.

[9] Kozłowski, G., Hereditary shape equivalences, (in preparation).

[10] Lacher, R.C., Cell-like mappings, I, Pacific J. Math., 30 (1969) pp. 717-731. 
[11] Mardešić, S. and Rushing T. B., Shape fibrations, I, General Top. and its Appl., 9 (1978) pp. 193-215.

[12] _ , Shape fibrations, II, Rocky Mountain J. Math., (to appear).

Institute of Mathematics

University of Tsukuba

Ibaraki, 305 Japan 